Research Journal of Applied Sciences, Engineering and Technology 6(20): 3838-3846, 2013

ISSN: 2040-7459; e-ISSN: 2040-7467

(C) Maxwell Scientific Organization, 2013

Submitted: January 17, 2013

Accepted: February 22, 2013

Published: November 10, 2013

\title{
Improvement in the Priority Handoff Scheme for Multi-Service Wireless Mobile Networks
}

\author{
${ }^{1}$ Cheng-Gang Liu, ${ }^{1}$ Zhen-Hong Jia, ${ }^{2}$ Xi-Zhong Qin, ${ }^{2}$ Lei Sheng and ${ }^{2}$ Li Chen \\ ${ }^{1}$ School of Information Science and Engineering, Xinjiang University, Urumqi 830046, China \\ ${ }^{2}$ Subsidiary Company of China Mobile in Xinjiang, Urumqi 830063, China
}

\begin{abstract}
In this study, a new handoff strategy to improve the performance of wireless mobile networks is presented. It has been found form this study that the dropping probability of handoff calls is drastically reduced compared to the existing method of channel reservation strategy of handoff calls and the performance of new calls can be improved. The strategy is that the new call should be delayed then it can use the last idle channel. The time that all the channels are holding has been shortened. Opportunities of the new call and handoff call occupancy channel are increased; the scheme also takes into account the priority of different data types and only when the highpriority data packets queue is empty, the data packets in low-priority data queue can be transmitted. Simulation results show that, to let the new voice call delay in the allowable range, can effectively reduce the dropping probability of handoff call and the blocking probability of high-priority data while improving the probability of new call to enter into the systems.
\end{abstract}

Keywords: Handoff, Markova process, multi-service, priority, queuing theory

\section{INTRODUCTION}

With the rapid development of mobile communication technology, support for multimedia applications in an increasing number of users has forced service providers to employ cells of smaller size so that availability of adequate capacity could be ascertained. Meanwhile, due to accelerate the moving speed of the users, the numbers of the users that need to handoff and the handoff during an ongoing call are increased continuously. Handoff failure that results in the forced termination of an ongoing call will add. Besides, the growth of the transmission bandwidth expanded the types of mobile communications service. In addition to voice services, data services such as Internet, image also appeared. In order to effectively use wireless resources and improve the system capacity and service QoS, designed handoff strategy that is suitable for voice, data and multi-service has been a hot research (Hamad et al., 2011; Sharna et al., 2011; Yuan-Yuan et al., 2010).

The grade of service of a wireless mobile network can be defined in terms of the blocking probability of new call and the dropping probability of handoff call.In handoff strategy, in order to improve the two indicators, two priorities are considered in many handoff strategies. Some handoff strategy taking into account different priorities between different service types have been proposed (Krishna et al., 2009; Al Khanjari et al., 2011; Suleiman et al., 2011), the different priorities between handoff calls and new calls other handoff are considered in other strategies. A new call being blocked is not as disastrous as a handoff call being dropped, so these strategies (Idil and Muhammed, 2007; Sheu et al., 2007; Nasr et al., 2010) consist of giving higher priority to handoff call than new call. At the same time, according to the different type of data service, consideration should also be given priority of different data types.

They (Krishna et al., 2009) proposed a movable boundary strategy; real time service has a higher priority than the non-real-time service. In order to increase the bandwidth utilization, non-real-time service is allowed to borrow unused channels from the real time service. Since handoff calls and new calls use the same set of channels, the dropping probability of handoff call is high when there are too many new calls. They (Idil and Muhammed, 2007) proposed the guard channel strategy that gives the handoff call a higher priority than a new call. This strategy consists of having a fixed number of channels in each cell, reserved exclusively for handoff call. It effectively reduces the dropping probability of handoff call. However, the drawback for using guard channel for handoff call is that the channels that are reserved may be unoccupied for a long duration and new calls are blocked because they have no channels can be used. They (Haw-Yun and Jean-Lien, 2004) proposed the guard channel strategy that consists of having a fixed number of channels in each cell, reserved exclusively for data call. 
When the arrival rate of voice services is high, voice services cannot occupy the guard channel of data services. This will result in a high blocking probability of voice service. They (Zhihua, 2008) have recently proposed a strategy to dynamically reserve guard channel for handoff calls. This increased the bandwidth utilization, but did not consider the different priorities between the various data services. They (Huang and Chan, 2004) proposed a movable boundary and guard channel strategy, they consider two priorities that real time service has a higher priority than the non-real-time service and the handoff call has a higher priority than a new call. The dropping probability of handoff call is significantly lower. However, the performance of new calls has been reduced and this strategy did not consider the different priorities between the various data services.

In the present study, a new handoff strategy based on multi-service is proposed. When services are busy in wireless mobile networks, handoff calls have a higher priority than new calls when call request. Idle channel can be assigned to handoff call immediately. If there is only one idle channel in the cell, new call would be delayed before it occupy the idle channel. Although the delay time of new calls are increased in new strategy, the probability of new call and handoff call to obtain a free channel are increased, the quality of voice service are improved.

In a data packet communication environment, data packets have different requirements. Some non realtime data packets can tolerate delays whereas real-time data packets are sensitive to delays. At the same time the strategy to provide differentiated services for data service can create more value for the communication operators. Combined with these characteristics of current data service in wireless mobile networks, new strategy takes different queue policies. According to the different importance of data services, data services are divided into high and low priority packet. For the smooth presentation of the idea of the new handoff strategy for performance improvement, the 4D Erlang theory with new handoff strategy is described. The reserved channel strategy and movable boundary channel strategy are compared with the new handoff strategy.

\section{SYSTEM MODEL}

The system model is shown in Fig. 1. In the following, we assume that the total number of channels in the channel pool shared by voice service and data service in each cell is fixed and denoted by C.N channels can be allocated to voice services and $C-N$ channels are allocated to data services. The voice calls are divided into handoff calls and new calls. Voice calls only can occupy the previous $\mathrm{N}$ channels. Handoff calls

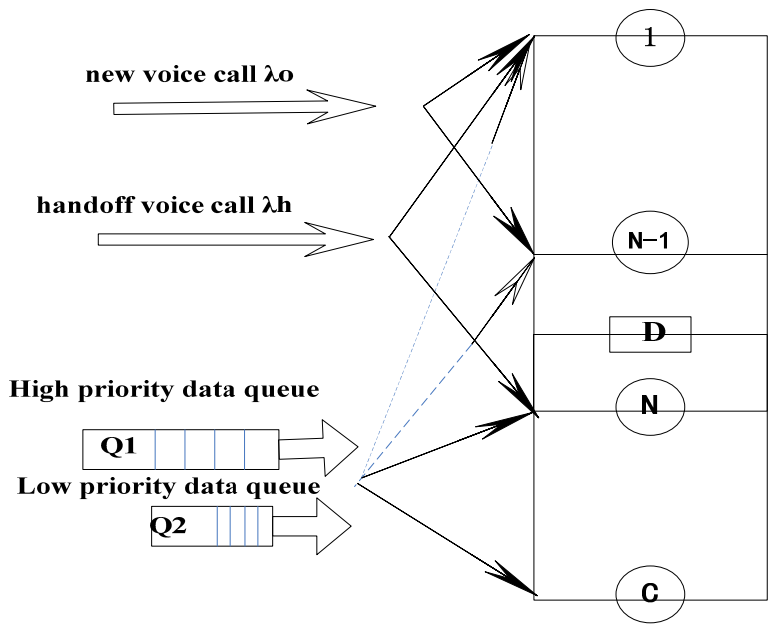

Fig. 1: System model

and new calls are endowed with the same priority in the previous $(N-1)$ channels.

Handoff calls are assigned priority over new calls when the $\mathrm{N}$-idle channel is allocated to voice call.

When the $\mathrm{N}$-th channel is idle in the cell, if the handoff call arrives, the $\mathrm{N}$-th channel can be directly occupied by the handoff call; if the new call arrives, the $\mathrm{N}$-th channel cannot be directly occupied by the new call, the new call would be delayed. During the delay time, if the users in the previous $(N-1)$ channels complete the call, then the idle channel in the previous $(N-1)$ channels can be occupied by the new call. If there is still no idle channel when the delay time is over, the $\mathrm{N}$-th idle channel can be occupied by new call.

According to the different importance of data services, data services are divided into high and low priority packet. When a data call arrives, if it is highpriority packet. It is stored in the queue $Q_{2}$. If it is a low-priority packet. It is stored in the queue $Q_{1}$. When the data packet is transmitted, the packet is removed from the queue. When the queue $Q_{2}$ is empty, the low priority packets can be transmitted in the queue $Q_{l}$. When the channel $(C-N)$ of the data service is busy, the channel assigned to the voice service in the idle time can be occupied by data services.

When a voice call arrives, an idle channel from $N$ channels can be assigned to the call. If there is no free channel can be occupied by voice call, while channels of the voice service are occupied by data services, the channel must be released immediately by the data call, the data call is placed in the queue $Q_{2}$ until there are the idle channels can be occupied. If the previous $(N-1)$ channels are occupied by all the voice calls, the voice call is treated differently according to the type of the call. In Fig. 1, The N-th channel can be occupied by handoff call immediately. The new call would be delayed. During the delay time, the system has been in a state $\mathrm{D}$. In the $\mathrm{D}$ state, there is at least one idle channel in the cell. The time that all channels are 
occupied is reduced. The time that the cell stays in the $(\mathrm{N}-1)$ state has been extended. Thereby the dropping probability of handoff call and the blocking probability of new call are reduced.

When the delay time $d(t)$ is over, if the users in the previous $(N-1)$ channels leave the cell, then the idle channel in the previous $(N-1)$ channels can be occupied by the new call. If there is not idle channel to be occupied in the previous $(N-1)$ channels, the N-th channel can be occupied by the new call.

\section{TRAFFIC MODEL}

This queuing system with $\mathrm{C}$ servers and an infinite number of users consisting of both voice and data service accords to Poisson process. The arrival rate of new calls is $\lambda_{o}$; the arrival rate of handoff calls is $\lambda_{h}$. Their service times are negative exponential distribution (Rivero-Angeles et al., 2009). Their service rate are $\mu_{o}$ and $\mu_{h}$. The maximum length of the data packets waiting queue is $Q_{1}$ and $Q_{2}\left(Q_{1}>Q_{2}\right)$, the arrangement rule is FIFO. The arrival rate of low priority packets and the arrival rate of high priority packets were $\lambda_{d l}$ and $\lambda_{d 2}$. The length of data packet is negative exponential distribution, the mean length of data packet is $L_{d}$ bits. Assuming that the transmission of data packet uses the same encoding, the transmission rate of data packet is $V_{d} \mathrm{kbit} / \mathrm{s}$ in the coding mode. The average transmission time of the low priority data packet and the high priority data packet is $1 / \mu_{d}=\mathrm{L}_{\mathrm{d}} / \mathrm{V}_{\mathrm{d}}$.

We represent the state of the cell with a fourdimensional Markov chain with states (i, j, l, g), where $i$ number of channels used by new calls in $\mathrm{N} ; j$ number of channels used by handoff calls in $\mathrm{N}$; $l$ number of high priority data calls in $Q_{2} ; g$ number of data calls in cell. The value ranges of discrete parameters are $\mathrm{i} \in[0, \mathrm{~N}]$, $\mathrm{j} \in[0, \mathrm{~N}], \mathrm{l} \in\left[0, \mathrm{Q}_{2}\right], \mathrm{g} \in\left[0, \mathrm{C}+\mathrm{Q}_{1}+\mathrm{Q}_{2}\right]$.

Since the steady-state distribution of the multidimensional continuous time Markov process is not solved easily and the actual system meets $\mu_{\mathrm{d}} /\left(\mu_{\mathrm{o}}+\mu_{\mathrm{h}}\right)$ $>>1$, the four-dimensional continuous time Markov process is decomposed into two consecutive twodimensional continuous time Markov process. The two parts are voice calls and data calls (Ghani and Schwartz, 1994).

Voice calls section: The state transition diagram of voice call is shown in Fig. 2. For $i$ and $j$ of State (i, j), $i$ is the number of new calls in the cell and $\mathrm{j}$ is the number of handoff calls in the cell. $\delta^{-1}=\mathrm{d}, \mathrm{d}$ is the delay time of new call. State D represents the state that the new call is delayed; the arrival rate to reach the state $\mathrm{D}$ is $\delta$. If a whole row is considered at the $y$-th position of a column, then the stationary probability states are given by the following equations:

$$
\begin{aligned}
& \lambda_{0} \pi_{(0, y)}=\mu_{0} \pi_{(1, y)} \\
& \left(\lambda_{0}+\mu_{0}\right) \pi_{(1, y)}=\lambda_{0} \pi_{(0, y)}+2 \mu_{0} \pi_{(2, y)} \\
& \left(\lambda_{0}+x \mu_{0}\right) \pi_{(x, y)}=\lambda_{0} \pi_{(x-1, y)}+(x+1) \mu_{0} \pi_{(x+1, y)}
\end{aligned}
$$

where, $\pi(\mathrm{x}, \mathrm{y})$ indicates the stationary probability of the state $(\mathrm{x}, \mathrm{y})$.

Let $\rho_{o}=\lambda_{0} / \mu_{0}, \rho_{o}$ is the call intensity of new call. Then we can think of the arrival of a new call is Poisson distribution parameters for $\rho_{o}$. The service time is negative exponential distribution parameters for 1 . Steady-state probability for each row of $N$ states (x, y) is:

$$
\pi_{(x, y)}=\frac{\rho_{0}^{x}}{x !} \pi_{(0, y)} \quad 0 \leq x \leq n-y, 0 \leq y \leq n-1
$$

If a whole column is considered at the $\mathrm{x}$-th position of a row, Let $\rho_{\mathrm{h}}=\lambda_{\mathrm{h}} / \mu_{\mathrm{h}}, \rho_{\mathrm{h}}$ is the call intensity of handoff call. Then we can think of the arrival of a handoff call is Poisson distribution parameters for $\rho_{h}$. The service time is negative exponential distribution parameters for 1. Steady-state probability for each column of $(\mathrm{N}+1)$ states $(\mathrm{x}, \mathrm{y})$ is:

$$
\pi_{(x, y)}=\frac{\rho_{h}^{y}}{y !} \pi_{(x, 0)} \quad 0 \leq x \leq n-y, 0 \leq y \leq n-1
$$

By substituting $\mathrm{x}=0$ in formula 5 and the resulting $\pi_{(0, y)}$ so obtained, if substituted in formula 4 , results in the following expression for the stationary probability states $\pi_{(\mathrm{x}, \mathrm{y})}$ :

$$
\pi_{(x, y)}=\frac{\rho_{0}^{x}}{x !} \frac{\rho_{h}^{y}}{y !} \pi_{(0,0)} \quad 0 \leq x \leq n-y, 0 \leq y \leq n-1
$$

In addition to (6), the following equations are derived for finding the remaining states $\pi_{(\mathrm{D}, \mathrm{y})}$ and:

$\pi_{(\mathrm{n}-\mathrm{y}, \mathrm{y})}$ :

$$
\begin{aligned}
& \left(\lambda_{0} / \mu_{0}+(n-y-1) \mu_{0}\right) \pi_{(n-y-1, y)}=\lambda_{0} \pi_{(n-y-2, y)} \\
& +(n-y-1) \mu_{0} \pi_{(D, y)}+(n-y) \mu_{0} \pi_{(n-y, y)} \\
& \left(\delta+(n-y-1) \mu_{0}\right) \mu_{0} \pi_{(D, y)}=\lambda_{0} \pi_{(n-y-1, y)} \\
& (n-y) \mu_{0} \pi_{(n-y, y)}=\delta \pi_{(D, y)}
\end{aligned}
$$

After solving the above equations, the following relations, the expressions for $\pi_{(\mathrm{D}, \mathrm{y})}$ and $\pi_{(\mathrm{n}-\mathrm{y}, \mathrm{y})}$ are obtained: 
Res. J. Appl. Sci. Eng. Technol., 6(20): 3838-3846, 2013

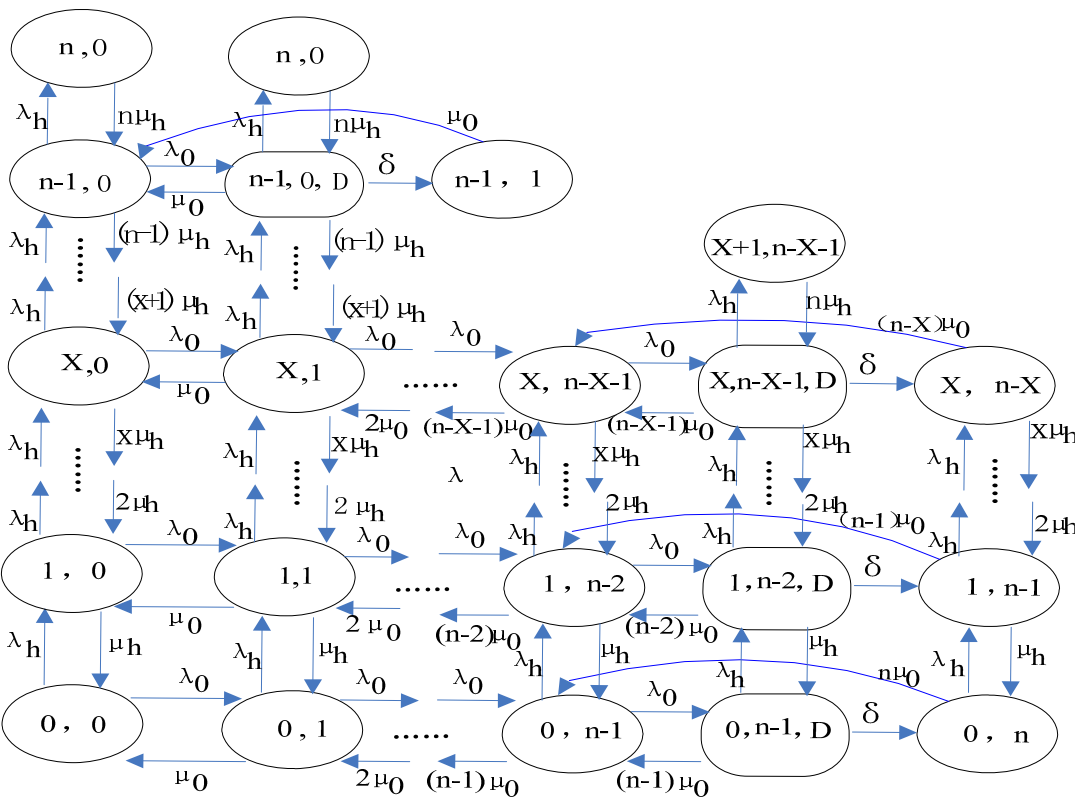

Fig. 2: State transition diagram of voice call

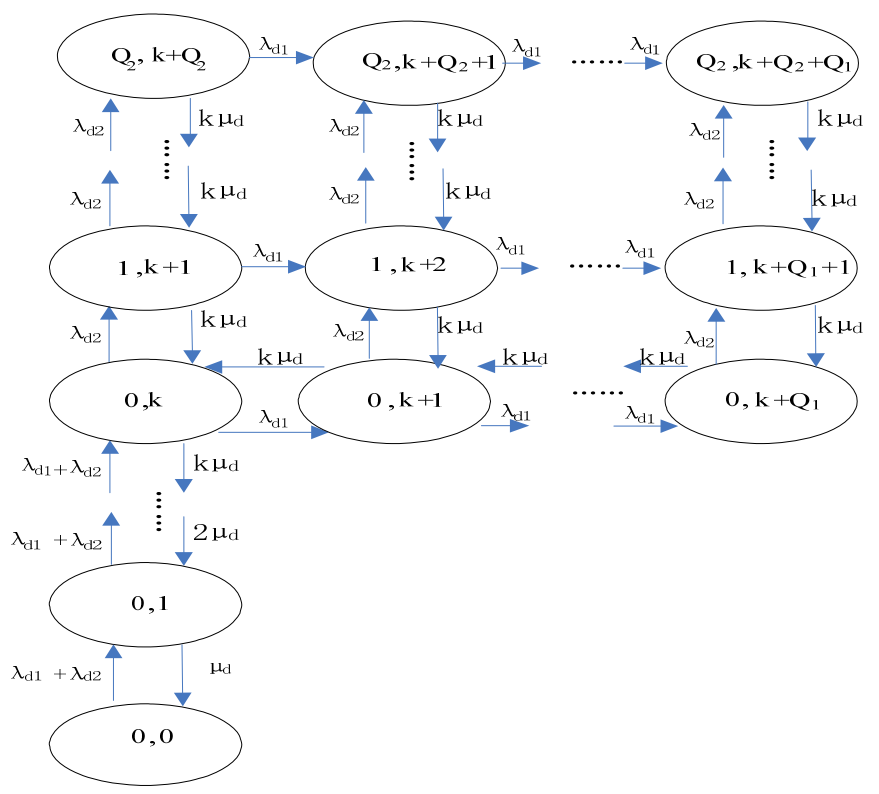

Fig. 3: State transition diagram of data call

$$
\begin{aligned}
& \pi_{(D, y)}=\frac{\lambda_{0}}{\delta+(n-y-1) \mu_{0}} \frac{\rho_{h}^{y}}{y !} \frac{\rho_{0}^{n-y-1}}{(n-y-1) !} \pi_{(0,0)} \\
& \pi_{(n-y, y)}=\frac{\delta}{\delta+(n-y-1) \mu_{0}} \frac{\rho_{h}^{y}}{y !} \frac{\rho_{0}^{n-y}}{(n-y) !} \pi_{(0,0)}
\end{aligned}
$$

where,

$$
\pi_{(0,0)}=\left(\sum_{y=0}^{n-1} \sum_{x=0}^{n-y} \frac{\rho_{h}^{y}}{y !} \frac{\rho_{h}^{x}}{x !}+Y\right)^{-1}
$$

$$
\begin{gathered}
Y=\sum_{y=0}^{n-1} \rho_{0} g_{y} \frac{\rho_{h}^{y}}{y !} \frac{\rho_{0}^{n-y-1}}{(n-y-1) !}+\sum_{y=0}^{n-1} \frac{\delta}{\mu_{0}} g_{y} \frac{\rho_{h}^{y}}{y !} \frac{\rho_{0}^{n-y}}{(n-y) !} \\
g_{y}=\frac{\mu_{0}}{\delta+(n-y-1) \mu_{0}}
\end{gathered}
$$

The dropping probability of handoff call in this case is:

$$
P_{d}=\sum_{y=0}^{n} \pi_{(n-y, y)}=\sum_{y=0}^{n} \frac{\delta}{\mu_{0}} g_{y} \frac{\rho_{h}^{y}}{y !} \frac{\rho_{0}^{n-y}}{(n-y) !} \pi_{(0,0)}
$$


And the blocking probability of new call is obtained as:

$$
\begin{aligned}
& P_{b}=\sum_{y=0}^{n-1} \pi_{(D, y)}+\sum_{y=0}^{n} \pi_{(n-y, y)} \\
& =\sum_{y=0}^{n-1} \rho_{0} g_{y} \frac{\rho_{h}^{y}}{y !} \frac{\rho_{0}^{n-y-1}}{(n-y-1) !} \pi_{(0,0)}+\sum_{y=0}^{n} \frac{\delta}{\mu_{0}} \frac{\rho_{h}^{y}}{y !} \frac{\rho_{0}^{n-y}}{(n-y) !} \pi_{(0,0)}
\end{aligned}
$$

Data calls section: When there are $\mathrm{w}(\mathrm{w}=\mathrm{i}+\mathrm{j})$ channels occupied by voice call in the system, the maximum of data calls simultaneously serviced is $\mathrm{k}$ $(\mathrm{k}=\mathrm{C}-\mathrm{w})$ in the system. For 1 and $\mathrm{g}$ of state $(\mathrm{l}, \mathrm{g}), \mathrm{l}$ is the number of the high priority packets in the queue $\mathrm{Q}_{2}$, $\mathrm{g}$ is the total number of packets in the system. The state transition diagram of data call is shown in Fig. 3.

Each state transition diagram of data call is described in Fig. 3, the principle of which is the flow conservation. Let inflow speed is equal to the outflow rate for each state. We can get the steady state balance equation:

$$
\begin{aligned}
& \left(\lambda_{d 1}+\lambda_{d 2}\right) \pi_{(l, g)}=(k+1) u_{d} \pi_{(l, g+1)} \quad 0 \leq g \leq k-l, l=0(17) \\
& \left(\left[1-u\left(g-k-Q_{1}\right)\right] \lambda_{d 1}+\left[1-u\left(l-Q_{2}\right)\right] \lambda_{d 2}+\right. \\
& \left.u(l-1) k u_{d}+\delta(l) \delta(g-k-1) k u_{d}\right) \pi_{(l, g)}= \\
& \lambda_{\mathrm{d} 1} \pi_{(g-1, l)}+u(l-1) \lambda_{\mathrm{d} 2} \pi_{(l-1, g-1)}+\delta(l) \delta(g-k) \lambda_{\mathrm{d} 2} \pi_{(l, g-1)} \\
& +u\left(Q_{2}-l-1\right) k u_{d} \pi_{(l+1, g+1)}+ \\
& \delta(l) u\left(k+Q_{1}-g-1\right) k u_{d} \pi_{(l, g+1)} \\
& k \leq g \leq k+Q_{1}+Q_{2}, 0 \leq l \leq Q_{2}
\end{aligned}
$$

where, $\pi(1, g)$ is the state transition probability of the system that is in state $(1, g)$. Let $\mathrm{u}(\mathrm{x})$ denote the step function, which is defined as follows: $\mathrm{u}(\mathrm{x})=1$ when $x \geq 0$ and $\mathrm{u}(\mathrm{x})=0$ when $x<0 ; \delta(\mathrm{x})=1$ when $x=0$ and $\delta$ $(\mathrm{x})=0$ when $\mathrm{x} \neq 0$.

For state equation of formula (15) and the formula (16), there are $\left(k+\left(\mathrm{Q}_{1}+1\right) \times\left(\mathrm{Q}_{2}+1\right)\right)$ equations. We coupled on the state probability normalized condition as formula (19); we can obtain the steady-state solution of state by solving linear equation:

$$
\sum_{g=0}^{k-1} \pi_{(0, g)}+\sum_{l=0}^{Q_{2}} \sum_{g=k+1}^{k+Q_{1}+Q_{2}} \pi_{(l, g)}=1
$$

The dropping probability of low priority data call in this case is:

$$
P_{d 1}=\sum_{l=0}^{Q_{2}} \pi_{\left(l, g+Q_{1}+l\right)}
$$

And the dropping probability of high priority data call is obtained as:

$$
P_{\mathrm{d} 2}=\sum_{g=k+Q_{2}}^{k+Q_{1}+Q_{2}} \pi_{\left(Q_{2}, g\right)}
$$

\section{SIMULATION RESULTS AND DISCUSSION}

For the numerical appreciation of the obtained results, the following parameters are assumed for a cell of a mobile cellular system: number of channels in the cell, $\mathrm{C}=14$, number of channels for voice services $\mathrm{N}=10$, high priority queue length $Q_{l}=200$, low priority queue length $Q_{2}=10$, average length of data packets, $L_{d}=12000$ bit. Assuming that the transmission of data packet uses the same encoding, the transmission rate of data packet is $\mathrm{V}_{\mathrm{d}}=9.05 \mathrm{kbits}$ in the coding mode. The arrival rate of new calls $\lambda_{o}=(1.34-8.04)$ $/ \mathrm{min}$, the arrival rate of handoff calls $\lambda_{h}=(1.66-5.0)$ $/ \mathrm{min}$. Their service rate are $\mu_{o}=0.67 / \mathrm{min}$ and $\mu_{h}=$ $0.83 / \mathrm{min}$. The arrival rate of low priority packets was $\lambda_{d l}$, the arrival rate of high priority packets was $\lambda_{d 2}$. $\lambda_{d l}=2 \lambda_{d 2}, \lambda_{d l}=0.45$ packets per second. Using MATLAB simulation software, when change the value of $\lambda_{0}, \lambda_{h}$, we can get the blocking probability of new call is $P_{b}$, the dropping probability of handoff call is $P_{d}$, the dropping probability of low priority data call is $P_{d l}$, the dropping probability of high priority data call is $P_{d 2}$.

Reserved channels for handoff calls, $r=3$ in the reserved channel strategy, number of channels for data calls in the cell, $m=4$. In the movable boundary channel strategy, the channel is not reserved for the handoff calls. When the voice dedicated channel is idle, the data call can occupy the voice channel, but when a voice user arrives, the data call must be released immediately channel. For ease of comparison, the new switching strategies proposed in this study and the protection of channel strategy and movable boundary policy, all consider the priority of different data types.

In Fig. 4 and 5, since new call would be delayed before it occupy the idle channel. The time of all channels were occupied is shorten, the time of the system in the state $(N-1)$ has been extended. The probability of new call and handoff call to obtain a free channel are increased. Compared with reserved channel strategy and movable boundary strategy, the dropping probability of handoff calls $P_{d}$ has been greatly improved in the new handoff strategy.

In Fig. 6 and 7, since there are not reserved channels in the new handoff strategy and movable boundary strategy, the new calls are less affected by handoff calls, there are smaller blocking rate in these two strategies than reserved strategy. The probability of an idle channel after a delay of a new call in the new 
Res. J. Appl. Sci. Eng. Technol., 6(20): 3838-3846, 2013

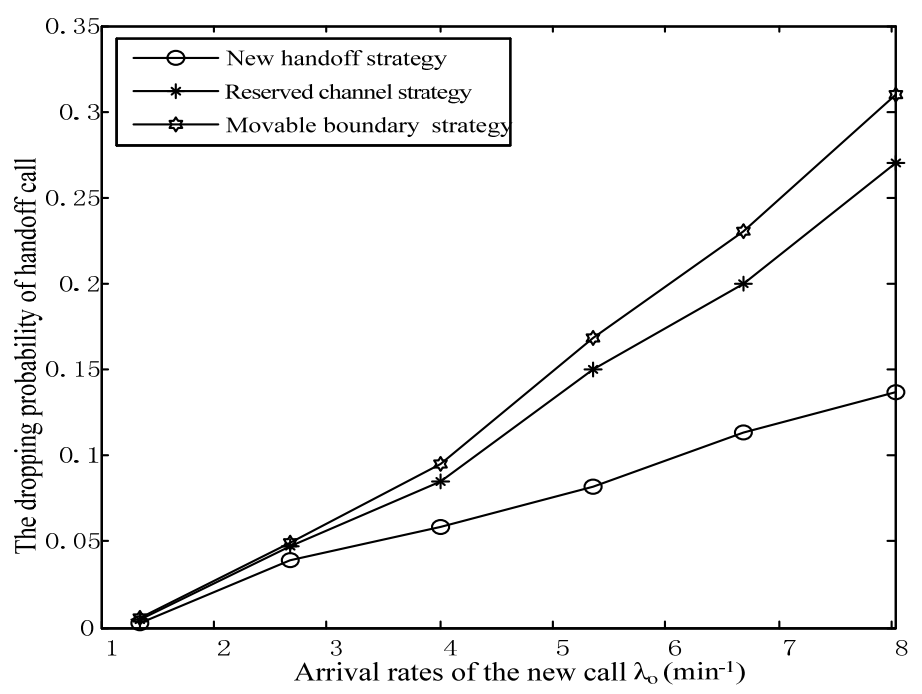

Fig. 4: The dropping probability of handoff call by arrival rates of the new call

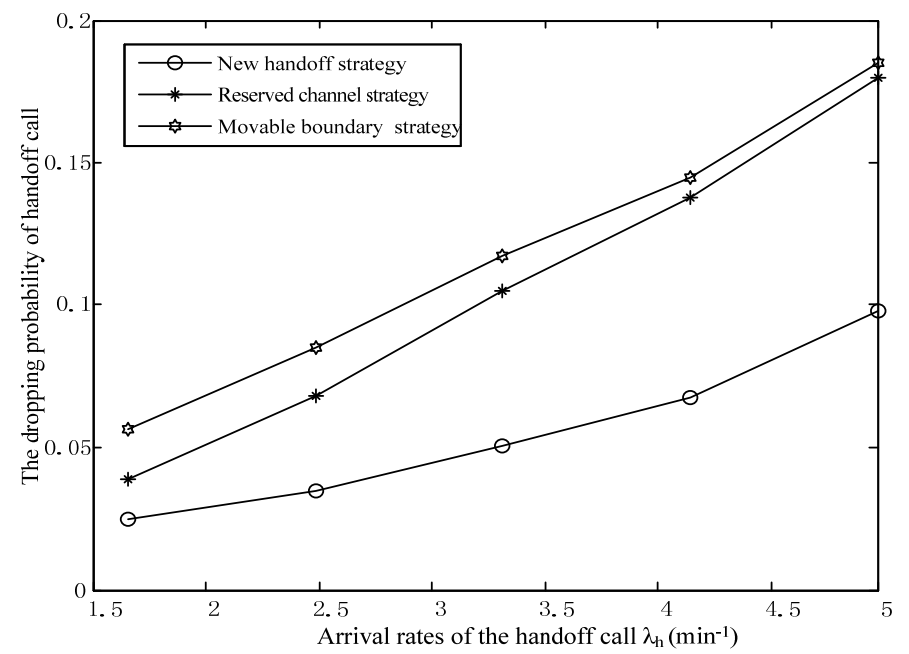

Fig. 5: The dropping probability of handoff call by arrival rates of the handoff call

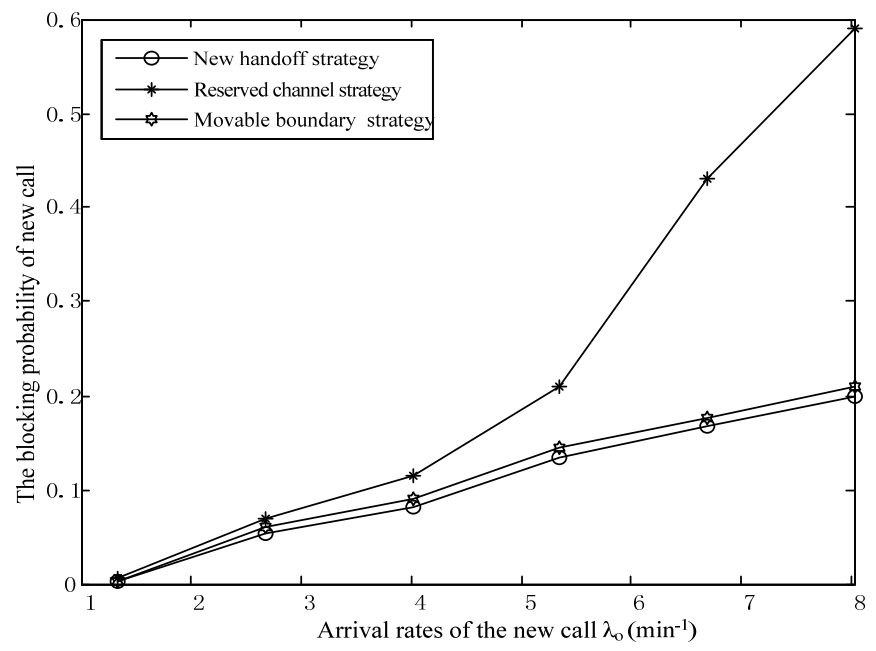

Fig. 6: The blocking probability of new call by arrival rates of the new call 
Res. J. Appl. Sci. Eng. Technol., 6(20): 3838-3846, 2013

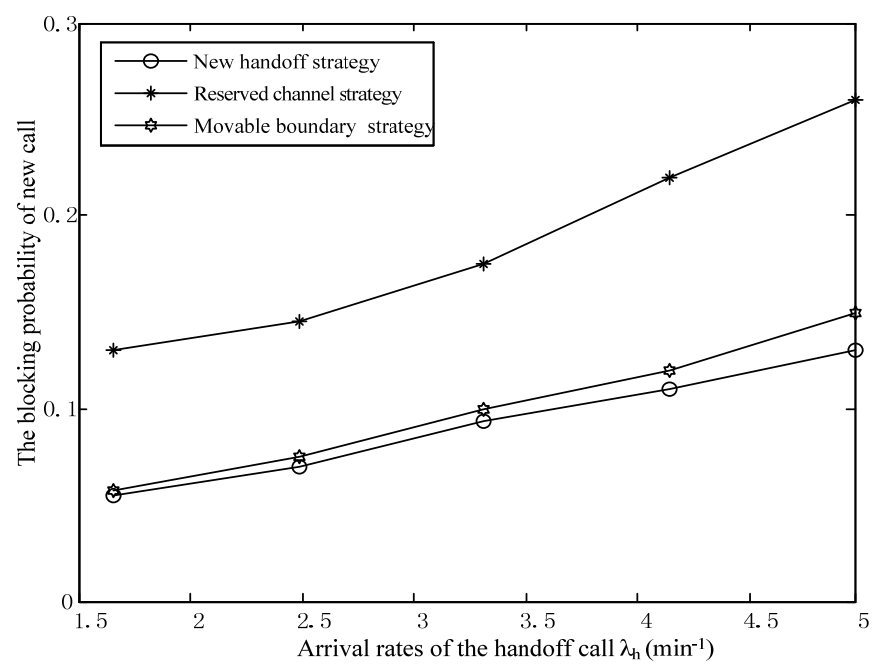

Fig. 7: The blocking probability of new call by arrival rates of the handoff call

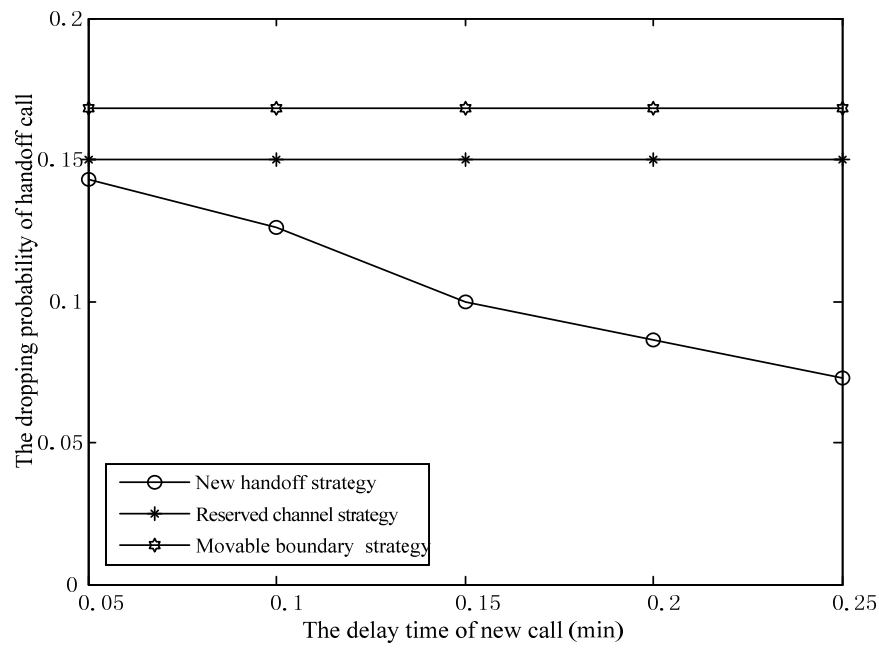

Fig. 8: The dropping probability of handoff call by the delay time of new call

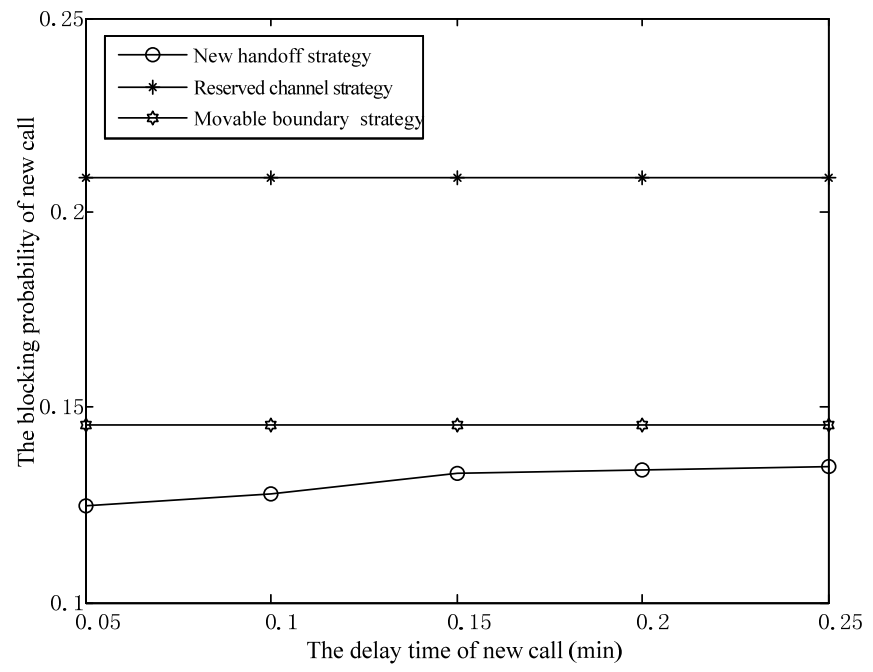

Fig. 9: The blocking probability of new call by the delay time of new call 


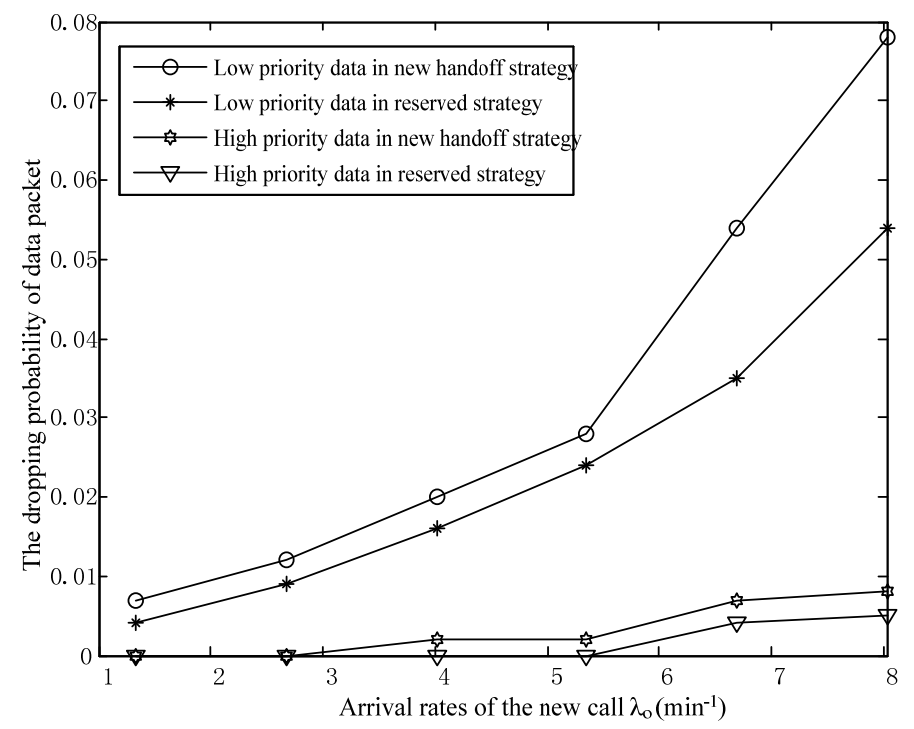

Fig. 10: The dropping probability of data packet by arrival rates of the new call

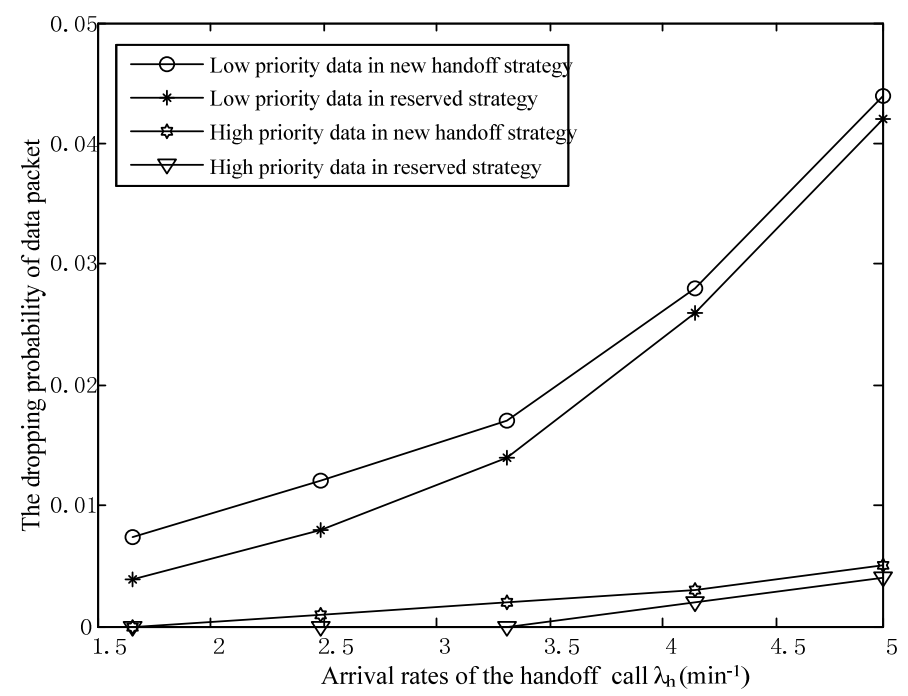

Fig. 11: The dropping probability of data packet by arrival rates of the handoff call

handoff strategy is greater than the movable boundary strategy, so the blocking probability of new call in the new handoff strategy is small than the movable boundary strategy.

In Fig. 8 and 9, the arrival rate of new calls $\lambda_{o}=5.358 / \mathrm{min}$, the arrival rate of handoff calls $\lambda_{h}=4.14 / \mathrm{min}$. Their service rate are $\mu_{o}=0.67 / \mathrm{min}$ and $\mu_{h}=0.83 / \mathrm{min}$. With the growth of the delay time of a new call, the probability of the idle channel will increase when the call arrives. Since the handoff calls are gave higher priority, an idle channel can be occupied by the handoff call immediately, the dropping probability of handoff calls decreased more significantly. Since the N- the idle channel is not allowed by the new calls, the blocking probability of new call is not changed significantly as the delay time increases.

In Fig. 10 and 11, the new handoff strategy is proposed that only when the high-priority data packets queue is empty, the data packets in low-priority data queue can be transmitted. Compared with the dropping probability of low priority data call $P_{d l}$, the dropping probability of high priority data call $P_{d 2}$ has been greatly improved.

Meanwhile, since the new call is not able to occupy the channel reserved for the handoff calls and data calls can borrow reserved channels in the reserved channels strategy, the dropping probability of data calls in the reserved channels strategy should be less than the new handoff strategy. However, the dropping probability of 
data calls will rapidly increase when the handoff call arrival rate is high.

\section{CONCLUSION}

A new handoff strategy based on business was presented in this study. In the actual communication process, users are more willing to accept occupies channel after the appropriate delay not directly blocked. A new call was allowed occupying idle channel after delay within the scope in this policy. The probability of the voice call into the system was increased. And the dropping probability of handoff call was reduced. The quality of service of a new call was improved at the same time. The dropping probability of high-priority data was reduced because it gives the data call a different priority. In order to reflect actual problems, we consider that the service time of handoff calls and new calls are different. New handoff strategy for improving network performance has certain significance.

\section{ACKNOWLEDGMENT}

This study is supported by 2012 Research and Development Fund Project of China Mobile Communications Group Co., Ltd. Xinjiang (Grant No. XJM2012-01).

\section{REFERENCES}

Al Khanjari, S., B. Arafeh, K. Day and N. Alzeidi, 2011. An adaptive bandwidth borrowing-based call admission control scheme for multi-class service wireless cellular networks. Proceeding of the International Conference on Innovationsin Information Technology (IIT), Abu Dhabi, pp: 375-380.

Ghani, S. and M. Schwartz, 1994. A decomposition approximation for the analysis of voice/data integration. IEEE T. Commun., 42(7): 2441-2452.

Hamad, A., M., Ehab and S. Adel, 2011. Performance analysis of a handoff scheme for two-tier cellular CDMA networks. Egypt. Inform. J., 12(2): 139-149.

Haw-Yun, S. and C.W. Jean-Lien, 2004. The study of dynamic multi-channel scheme with channel deallocation in wireless networks. Comput. Netw., 45(4): 463-482.

Huang, Q. and S. Chan, 2004. An enhanced handoff control scheme for multimedia traffic in cellular networks. IEEE Comm. Lett., 8(3): 195-197.

Idil, C. and S. Muhammed, 2007. Analytical modeling of a time-threshold based bandwidth allocation scheme for cellular networks. Comput. Commun., 30(5): 1036-1043.
Krishna, P.V., S. Misra, M.S. Obaidat and V. Saritha, 2009. An efficient approach for distributed dynamic channel allocation with queues for realtime and non-real-time traffic in cellular networks. J. Syst. Softw., 82(7): 1112-1124.

Nasr, A.A., H.M. El-Badawy and H.M. El-Hennawy, 2010. Connection admission control reward optimization for different priority classes in homogeneous wireless network. Proceeding of the 6th International Conference on Wireless and Mobile Communications (ICWMC), Valencia, 2010: 549-555.

Rivero-Angeles, M.E., D. Lara-Rodriguez and F.A. Cruz-Perez, 2009. Differentiated back off strategies for prioritized random access delay in multiservice cellular networks. IEEE T. Veh. Technol., 58(1): 381-397.

Sharna, S.A., M.R. Amin and M. Murshed, 2011. Call admission control policy for multiclass traffic in heterogeneous wireless networks. Proceeding of the 11th International Symposium on Communications and Information Technologies (ISCIT), Hangzhou, 2011: 433-438.

Sheu, T.L., Y.J. Wu and B. Li, 2007. A generalized channel preemption model for multiclass traffic in mobile wireless networks. IEEE T. Veh. Technol., 56(5): 2723-2732.

Suleiman, K.H., H.A. Chan and M.E. Dlodlo, 2011. Analysis of serving discipline algorithms for cellular networks. Proceeding of the 2nd International Conference on Wireless Communication, Vehicular Technology, Information Theory and Aerospace \& Electronic Systems Technology (Wireless VITAE), Chennai, 2011: 1-6.

Yuan-Yuan, S., W. Ren-Yong, L. Ren-Fa, D. Wei and L. Jian-Hui, 2010. A novel measurement-based call admission control algorithm for wireless mobility networks under practical mobility model. Proceeding of the International Conference on Communications and Mobile Computing (CMC), Shenzhen, 3: 364-368.

Zhihua, Z., 2008. Performance analysis of a QoS guaranteed dynamic channel reservation for handoff prioritization in cellular mobile networks. Proceeding of the 4th International Conference on Wireless Communications (WICOM'08), USA, 2008: 1-4. 